\title{
Efectos de la formación universitaria del gerente de pymes familiares en la motivación laboral del empleado
}

\section{The effect of CEO's education level of family SME in the degree of work motivation of employees}

\author{
Antonio José Carrasco Hernández ${ }^{\mathrm{a}}$ Ángel Luis Meroño Cerdan ${ }^{\mathrm{b}, *}$
}

a, bepartamento de Organización de Empresas y Finanzas, Universidad de Murcia, Facultad de Economía, Campus de Espinardo, Murcia, 30100 (Spain)

\author{
Historial: \\ Recibido 30-06-2010 \\ Aceptado 21-12-2010 \\ Palabras clave: \\ Empresa familiar \\ Gerente \\ Motivación \\ Desempeño \\ Códigos JEL: \\ M53, L25
}

D A T O S A R TÍC U L O

\begin{abstract}
R E S U M E N
El presente trabajo, analiza el efecto de la formación del gerente en el grado de motivación laboral de los empleados, así como, su efecto en el desarrollo de ventajas competitivas sostenibles en la pyme familiar. El estudio empírico se desarrolla sobre una muestra de 396 pyme familiares españolas y se encuentra que el potencial motivador del puesto varía en función de la formación del gerente. La motivación en el puesto es mayor, en organizaciones familiares con un gerente formado, al incrementarse el grado de autonomía, la retroalimentación y la significación del trabajo para el empleado. Por último, el rendimiento de la organización es mayor al aumentar la formación del gerente y el grado de motivación del trabajo, lo que confirma los argumentos de la teoría de recursos y capacidades aplicados a la pyme familiar.
\end{abstract}

\author{
A R T I C L E IN F O \\ Article history: \\ Received 30 June 2010 \\ Accepted 21 December 2010 \\ Keywords: \\ Family Business \\ CEO \\ Motivation \\ Performance \\ JEL codes: \\ M53; L25
}

\begin{abstract}
A B S T R A C T
The role of $\mathrm{CEO}$ is essential in family business. Numerous investigations have shown a positive relationship between the improvement of the company and the amount of training manager. No such relationship has been examined for the degree of work motivation. Therefore, in this study was to evaluate the effect of the CEO's education level in the degree of work motivation of employees, as well as its effect on the development of sustainable competitive advantages in the SME family.

The empirical study found that the motivating potential of the post varies according to CEO's education level. Thus, the motivation on the job is higher in family business with a manager with high training, when increasing the degree of autonomy, feedback, and the significance of work for the employee. Finally, the performance of the organization is greater, increasing the training manager and the motivation of the work
\end{abstract}

\footnotetext{
Autor de contacto. Tel.: +34968363791

Correos electrónicos: antonioc@um.es,angelmer@um.es
} 


\section{Introducción}

Actualmente, las organizaciones familiares se enfrentan a una situación contextual compleja, acentuada por el debilitamiento de las principales economías mundiales, el aumento del desempleo, la pérdida de capacidad de consumo en las familias, y la creciente competencia internacional. En este nuevo contexto, las pyme familiares han de optimizar la utilización de sus recursos y ser más competitivas, identificando y explotando las oportunidades que les ofrece el entorno (Hitt, Ireland, Camp y Sexton, 2001, 2002).

Un marco teórico apropiado, desde el que abordar el análisis del desempeño organizativo, desde un escenario competitivo, a partir del análisis de los recursos de la empresa, es la teoría de recursos y capacidades (Barney, 1986). Con esta teoría se afirma que la heterogeneidad de desempeños entre empresas puede explicarse por la capacidad de cada una de ellas para obtener ventajas sobre la competencia, y de la habilidad para identificar, desarrollar, proteger y aprovechar los activos estratégicos empresariales, en este caso con una implicación mejor y una utilización más adecuada de los recursos humanos. De esta manera, se alcanzarán ventajas competitivas sostenibles, capaces de apropiarse de las rentas producidas y de promover en la organización un mayor éxito.

Los recursos generados por la interacción entre la familia y la empresa (Edleston, Kellermanns y Sarathy, 2008; Habbershon, Williams y MacMillan, 2003), son los que diferencian y desarrollan ventajas competitivas sostenibles, o desventajas competitivas, en la pyme familiar, por lo que merecen especial atención (Makadok, 2001; Kellermanns, 2005). En el desarrollo de estos recursos, el papel del gerente es clave, al ser generalmente, miembro de la familia, en un porcentaje muy alto de ocasiones (Monreal et al., 2009), y al tener sus decisiones un gran impacto en los recursos y miembros de la pyme. Es por ello, que merece una especial mención y atención en los estudios de competitividad en las pyme familiares.
Por otro lado, en los últimos años, es común escuchar que una parte de la competitividad de las empresas reside en el bienestar de sus trabajadores. La experiencia viene demostrando que el éxito de los empleados en el desempeño de sus funciones viene dado, según el grado de motivación laboral, fruto de la adaptación del empleado a su puesto de trabajo. Por ello, el estudio del puesto es clave para el desarrollo de ventajas competitivas organizativas.

En este sentido, Fernandez (2004) señala que para que los trabajadores estén motivados y trabajen a pleno rendimiento, han de estar cómodos en su puesto de trabajo. Además, han de estar motivados para poder ser lo más productivos para la organización. Por lo tanto, el desarrollo de capacidades organizativas, parte de la adecuada adaptación y alineación de los empleados con sus puestos. El puesto es la esencia misma del grado de productividad de una empresa consistente en un grupo de tareas a desarrollar para que la organización puede lograr sus objetivos (Noe, 1997).

Las características del puesto son las que nos determinan el grado de motivación laboral. Así, a partir de modelos como los de Hackman y Oldman (1976), se determina que la motivación laboral aumenta al mejorar las características del puesto. Un diseño deficiente de tales características es fuente de desmotivación, insatisfacción y baja productividad de los recursos humanos (en adelante RR.HH.) (Malik, 2000).

No obstante, que la mayoría de los trabajos se centre en el estudio de las características del puesto, o en la medición de sus efectos, en grandes empresas, contrasta con el hecho de que muchas investigaciones han demostrado que las prácticas de recursos humanos de las pymes difieren de las de otras empresas (De Kok et al., 2006). Esto es más acusado si consideramos, en especial, el caso de las pymes familiares, que presentan unas peculiaridades organizativas que las diferencian de cualquier otro tipo de organización, sobre todo en la forma en la que aplican las prácticas de recursos humanos (De Kok et al., 2006).

Carrasco Hernández, A. J. y Meroño Cerdan, A. L. (2011). Efectos de la formación universitaria del gerente de pymes familiares en la motivación laboral del empleado. Revista de Empresa Familiar, 1(1), pag. 35-51. 
De lo anterior se deduce que es preciso incrementar los estudios en esta materia (Reid y Adams, 2001). Se precisan nuevos estudios, desde los que se examine, el diseño de puestos en la pyme familiar. También, desde los que se evalué los efectos de la familia en el diseño de los puestos. Un miembro importante de la familia en la empresa es el gerente, ya que es el factor más importante en la pyme familiar. Así, con esta investigación, se analiza el efecto de la formación del gerente en el grado de motivación laboral de los empleados, así como, su efecto en el desarrollo de ventajas competitivas sostenibles en la pyme familiar.

Las razones que nos han llevado a seleccionar sólo pymes familiares y descartar otras son diversas. En primer lugar, es una ventaja estudiar sólo a un grupo de organizaciones, entre las que existe mayor homogeneidad. Al menos se alcanzan unos resultados más análogos y generalizables para estas organizaciones. En segundo lugar, son organizaciones que presentan una configuración especial en su propiedad y dirección, configuraciones que afectan a las prácticas de recursos humanos desarrolladas (De Kok et al., 2006). Por ello, controlar en este estudio dichos elementos nos permitirá identificar mejor los efectos de la formación del gerente en la motivación laboral en la empresa. En tercer lugar, las pymes de carácter familiar son organizaciones que merecen una especial consideración, al ser altamente importantes en el desarrollo económico y social de cualquier área económica. Concretamente, este tipo de empresa representa más del $80 \%$ del tejido empresarial español, lo que las convierte en pieza clave de la creación de empleo y riqueza (Monreal et al., 2009). En cuarto lugar, incrementar el número de investigaciones de recursos humanos en las pymes familiares acercaría la importancia real a la científica y contribuiría a mejorar ese conocimiento existente sobre tales organizaciones. Así, intentamos ayudar a los empresarios de dichas empresas para que valoren la importancia de su formación universitaria en la empresa, y que el esfuerzo de obtener una cualificación en la empresa resulta necesario e importante para afrontar el nuevo escenario empresarial al que se enfrentan las pymes. Por último, y para comprobar si los argumentos de la teoría de recursos y capacidades sirven para explicar las prácticas de recursos humanos en las pymes familiares, esta teoría se ha aplicado escasamente hasta el momento al estudio de tales empresas.

Para cumplir estos objetivos, el trabajo se ha estructurado de forma tradicional. En primer lugar, se identifica el concepto de pyme familiar. A continuación, se evalúan los factores que influyen en la motivación laboral, en concreto, el efecto de la formación del gerente en la motivación laboral de los empleados. Posteriormente, se presenta la metodología del estudio, con la descripción de la población, muestra y variables, así como los análisis y resultados. Y por último las conclusiones, limitaciones y líneas futuras del trabajo.

\section{Motivación laboral de los empleados y formación del gerente. Un enfoque de recursos y capacidades}

Existen diferentes marcos teóricos desde los que explicar las diferencias entre empresas familiares, aunque, no todos sirven para esclarecer cómo los recursos de la empresa producen dichas diferencias, ni cuándo, ni por qué, se producen, ni qué efectos ocasionan en el desempeño de la organización (Chrisman et al., 2005; Habbershon y Williams, 1999). La teoría de recursos y capacidades se muestra como un enfoque apropiado para explicar, desde un escenario competitivo, cómo los recursos de la empresa familiar producen ventajas competitivas sostenibles, tras ser combinados entre sí (Cabrera-Suarez et al., 2001; Habbershon y Williams, 1999). También, para aclarar cuándo, cómo y por qué tales ventajas producen mayores desempeños organizativos (Dyer, 2006; Chrisman et al., 2005; Habbershon y Williams, 1999).

En el enfoque de recursos y capacidades se sugiere que las empresas sobreviven cuando son capaces de combinar recursos que son imperfectamente movibles $y$ heterogéneos (Wernerfelt, 1984; Penrose, 1959). De forma 
amplia, se incluyen entre aquellos recursos a los que convierten a las empresas en complejas, idiosincráticas y únicas (Barney, 1991), procedentes de procesos internos y activos intangibles, además de los valores, creencias, símbolos e interrelaciones, establecidos por los individuos que forman la empresa (Barney, 1991).

La teoría de recursos y capacidades asume que existe heterogeneidad entre empresas, ya que cada organización es diferente, en función de su dotación de recursos, y sobre éstos se sustentan las ventajas competitivas.

Por otro lado, esta teoría se basa en la sostenibilidad de las rentas, ya que la heterogeneidad puede o no persistir en el tiempo, por lo que, para hablar de ventaja competitiva sostenible, las empresas deben mantener rentas superiores en el largo plazo, y no todos los recursos son susceptibles de lograr ventajas competitivas sostenibles, sino tan sólo aquellos que son valiosos, escasos, imperfectamente imitables, y no sustituibles (Barney, 1991), y que duran en el tiempo (Amit, y Schoemaker, 1993), y que adecuadamente combinados con otros recursos, generan capacidades diferenciadoras en la empresa.

Son los recursos de carácter intangible, los más valiosos, escasos, inimitables y no sustituibles para la organización. Wernerfelt (1984) define recurso como "cualquier cosa que pueda considerarse una fortaleza o una debilidad en una empresa y como aquellos activos tangibles e intangibles que están relacionados de manera semipermanente con la empresa".

Dentro de los recursos de la empresa cabe destacar la importancia de los recursos humanos como factor crítico de éxito (Habbershon et al., 2003). Son numerosos los trabajos que han mostrado que estamos ante un recurso capaz de añadir valor al proceso productivo, caracterizado por su escasez y durabilidad (Barney y Wright, 1998), por lo que se puede considerar como fuente de ventaja competitiva sostenible y favorecer el éxito de la organización familiar (Habbershon et al., 2003).
De entre los recursos humanos, cabe destacar al gerente, en concreto, su capacidad directiva, ya que con sus decisiones se encontrará o no, el perfecto alineamiento entre los recursos de la empresa, y el desarrollo de ventajas competitivas sostenibles (Schein, 1982).

Entender el alineamiento de los recursos en la pyme familiar implica, además, examinar la implicación de la familia, en la dirección y/o propiedad de la empresa (Priem y Butler, 2001; Barney y Arikan, 2001; Mahoney, 1995), entender cómo a partir de la implicación de la familia, se generan recursos únicos, frente a otras organizaciones (Chrisman et al., 2005; Habbershon y Williams, 1999).

En concreto, recursos diferenciadores fruto del efecto ocasionado por la implicación de la familia en las estructuras de poder del negocio (Klein et al., 2005), siendo las capacidades directivas de la familia un factor importante para examinar en este sentido. Capacidades directivas que en el caso de la pyme familiar se materializan en la figura del gerente, dado el reducido número de directivos en estas empresas.

La pyme en todo momento necesita gerentes que posean destrezas y habilidades con las que estimular, comprometer y hacer participar a los empleados en los distintos niveles de trabajo. En las funciones del gerente esta planificar y organizar el trabajo en la empresa, asignar e integrar a los empleados y medir los resultados del proceso (Drucker, 2002).

Las decisiones gerenciales correctas, reportarán ventajas competitivas a las pyme si son capaces de aprovechar (Rubio y Aragon, 2009): a) el buen clima laboral existente, motivado por los excelentes canales de comunicación, b) los mayores niveles de flexibilidad, c) la alta motivación laboral, al permitir a los trabajadores observar con facilidad la relación entre las tareas desarrolladas en los puestos y el resultado final obtenido por la empresa, y ofrecer al mismo tiempo puestos de trabajo variados y d) la mayor cercanía del directivo a los puestos de trabajo. 
El gerente es la persona que adopta las decisiones generales de la empresa, planifica los objetivos a conseguir y diseña los medios para alcanzarlos, aunque después delegue las decisiones en niveles inferiores (Rubio, 2004). $\mathrm{Su}$ papel crucial está fuera de toda duda en cualquier tipo de empresa, sin embargo su importancia en las pyme es, si cabe, aún más importante.

En los directivos de las pyme suele recaer la responsabilidad de elegir entre distintas opciones estratégicas, incluso suelen ser ellos los que implementan las tácticas de negocio y gestionan la mayoría de sus recursos. Por ello, se les califica como el activo más importante (Lerner y Almor, 2002), y la falta de capacidad directiva se enumera como una de las principales causas de fracaso en las pyme (Martin y Staines, 1994; Ahire y Golhar, 1996).

El nivel de educación, al ser un reflejo de los conocimientos y habilidades poseídos, se relaciona de forma positiva: con la capacidad del directivo para realizar elecciones estratégicas de acuerdo con las exigencias del entorno (Wiersema y Bantel, 1992), con su propensión a generar e implementar soluciones creativas a los problemas (Bantel y Jackson, 1989), e, incluso, con el nivel de productividad de la empresa (Norburn y Birley, 1988). Asimismo, algunos trabajos evidencian que existe una relación positiva entre el éxito y el nivel de educación de los directivos (Martin y Staines, 1994). Esto sugiere que las empresas que cuenten con directivos con un mayor nivel de formación alcanzarán mayores niveles de desempeño.

Y si entendemos el desempeño, cómo una mayor excelencia empresarial, esto es, mejores resultados empresariales, mayor excelencia laboral, fruto de unos niveles más de motivación laboral, cabe esperar que:

$\mathrm{H}_{1}$ : La motivación laboral de los empleados aumente al incrementarse la formación del gerente.

$\mathrm{H}_{2}$ : La formación del gerente modere positivamente la relación entre la motivación laboral de los empleados y el desempeño organizativo.

\section{Metodología y diseño de la investigación.}

\subsection{Población y muestra}

A la hora de abordar cualquier investigación sobre empresa familiar es preciso ofrecer una definición (Monreal et al., 2009). Existen múltiples definiciones de empresa familiar, de mayor o menor rigor académico, así como más o menos complejas metodológicamente. Intuitivamente todos tenemos una idea concreta sobre las particularidades de este tipo de empresas.

De las múltiples definiciones existentes, hemos adoptado una sencilla, generalmente aceptada, neutra o intermedia.

Para ello, nos hemos basado en el estudio de Shanker y Astrachan (2003), en el que se define a la empresa familiar como aquella en la que la propiedad y la gestión esta, en manos del fundador/es o sus descendientes. A partir de aquí surgen las discusiones sobre cuál debe ser el porcentaje en la propiedad, cuál en la gestión y si la continuidad es simplemente intencional o de hecho supone la incorporación de segundas o ulteriores generaciones.

Después de numerosas disquisiciones teóricas, a mediados de 2008, las principales instituciones representativas de las empresas familiares han llegado a una definición oficial. Tanto el Grupo Europeo de Empresas Familiares (GEEF) como el Family Business Network (FBN) han convenido, con una finalidad operativa, relacionada con la promoción y las ayudas europeas destinadas para este tipo de empresas, que familiar es una compañía, tenga el tamaño que tenga, en la que la mayoría de los votos son propiedad de la persona o personas de la familia que fundó o fundaron la compañía y al menos un representante de la familia o pariente participa en la gestión o gobierno de la compañía.

A partir de estas definiciones surgen diversas estadísticas, el propio IEF estima que hay más de 2,9 millones y medio de empresas familiares en España. Siendo un $85 \%$ de las empresas españolas familiar. En términos de empleo 
representan el 75\% del empleo privado, es decir, dan empleo a más de 13,9 millones de trabajadores. El total de su facturación equivale al $70 \%$ del PIB español.

El estudio empírico se ha desarrollado sobre una población de 3.413 pyme familiares, procedentes del sector industrial y servicios de la Región de Murcia, y extraídas de una población total de 5.251 empresas, familiares y no familiares. Se ha utilizado el trabajo de Monreal et al. (2009) para estimar la población de las empresas familiares en la Región de Murcia.

Para obtener la población de empresas y la muestra, utilizamos la base de datos SABI (Sistema de análisis de balances ibéricos), en la que se contienen datos de empresas de españolas.

De dicha base se han seleccionado aquellas empresas de más de 10 trabajadores, y de menos de 250 trabajadores. Se han descartado a las empresas de menor tamaño, por su falta de formalización, así como por no tener un conjunto de prácticas organizativas claramente predefinidas. También, se han descartado a empresas de gran tamaño, por existir gran diferencia de recursos entre las mismas, y las empresas de pequeño y mediano tamaño. Este mismo procedimiento de descarte, se ha realizado en otros trabajos de empresa familiar (ver Fernández y Nieto, 2005 y 2006; y Davis y Haverston, 2000).

La población de empresas obtenida, se han comparado para examinar su coincidencia con la de otras bases de datos como D.I.R.C.E (publicada por el Instituto Nacional de Estadística - http://www.ine.es). Y partiendo de la población de empresas total identificada, se estimo el tamaño mínimo muestral.

Tomando esta restricción, se ha obtenido que para un nivel de confianza del $95,5 \%$, un error del $5 \%$ y $\mathrm{p}=\mathrm{q}=0.5$, se necesita una muestra mínima del 358 empresas familiares, en la población de 3.413 empresas familiares estimadas. Es decir, se deben examinar, partiendo que las empresas familiares son el $65 \%$ de la muestra (ver tabla 1), 550 empresas en total.

\section{Tabla 1}

Población de pyme familiar en la Región de Murcia por tamaño.

\begin{tabular}{lccl}
\hline & Total & $\begin{array}{c}\text { Familiares Familiares } \\
(\%)\end{array}$ \\
\hline $\begin{array}{l}\text { 10 a 19 3.051 } \\
\text { empleados }\end{array}$ & $68,6 \%$ & 2.093 \\
$\begin{array}{l}\text { 20 a 49 1.601 } \\
\text { empleados }\end{array}$ & $64,6 \%$ & 1.034 \\
$\begin{array}{l}50 \text { a } 99 \\
\text { empleados }\end{array}$ & 536 & 208 \\
$\begin{array}{l}\text { 100 hasta } 213 \\
\text { 250 39,9\% }\end{array}$ & 85 \\
empleados & & \\
\hline TOTAL 5.251 & $65,00 \%$ & 3.413 \\
\hline
\end{tabular}

Fuente: Elaborado a partir de Monreal et al. (2009)

Las empresas seleccionadas en la muestra son representativas, del tejido empresarial murciano, por tamaño y sector de actividad. Se ha partido de una selección de muestra, de mayor exigencia, y se ha seleccionado una muestra inicial de 800 empresas sobre las 5.251, y se han examinado, en la muestra, empresas familiares y no familiares. La razón de no estudiar en la muestra inicialmente, a empresas familiares sólo, es que no existe una base de datos poblacional que te identifique al $100 \%$, qué empresas son familiares y qué empresas no lo son.

La realización del estudio se basará en información primaria obtenida directamente de las empresas. Dicha información será recogida mediante la realización de encuestas personales contestadas únicamente por el gerente, sea o no familiar. Con esta metodología se trata de asegurar la mayor calidad y fiabilidad posible de los datos. El cuestionario que ha sido utilizado para la realización de entrevistas está compuesto de preguntas cerradas, las cuales han sido diseñadas, a partir, de un análisis minucioso de la literatura investigadora. Se recogieron 554 cuestionarios válidos (de los 800 lanzados), lo que representa una tasa de respuesta del $69,25 \%$, y un error muestral total del $4,01 \%$, para un nivel de confianza del $95,5 \%$ y $p=q=0.5$. Esto implica que la muestra obtenida es altamente representativa de la población y muestra total seleccionada. 
En la muestra se identificaron 385 pyme familiares, muestra superior a la mínima necesaria.

\section{Tabla 2}

Muestra de empresas familiares por tamaño

\begin{tabular}{lccc}
\hline Empleados & $\begin{array}{c}\text { Población } \\
\text { EF }\end{array}$ & $\begin{array}{c}\text { Muestra } \\
\text { EF }\end{array}$ & $\begin{array}{c}\text { Error } \\
\text { muestral }\end{array}$ \\
\hline 10 a 19 & 2.093 & 156 & \\
20 a 49 & 1.034 & 153 & \\
50 a 250 & 293 & 75 & \\
\hline TOTAL & 3.413 & 385 & $4,80 \%$ \\
\hline
\end{tabular}

Fuente: Elaboración propia

En la tabla 2 se muestra información de la representatividad de la muestra de empresa familiar por tamaño. Atendiendo a los criterios de tamaño y actividad, en la Región de Murcia se estiman aproximadamente 3.413 empresas familiares, atendiendo a las estimaciones de Monreal et al. (2009). Considerando la estimación para cada intervalo de la presencia de empresas familiares se ha determinado la columna de población de empresas familiares (Población EF) para cada intervalo de tamaño. Junto con la información de la muestra permite calcular el error muestral total, la proporción de empresas familiares, para población y muestra, para cada intervalo de tamaño.

\section{Tabla 3}

Muestra de empresas familiares por sectores.

\begin{tabular}{lccc}
\hline Actividad & $\begin{array}{c}\text { Población } \\
\text { EF* }\end{array}$ & $\begin{array}{c}\text { Muestra } \\
\text { EF }\end{array}$ & $\begin{array}{c}\text { Error } \\
\text { muestral }\end{array}$ \\
\hline Industria & 883 & 104 & \\
$\begin{array}{l}\text { Construcción } \\
\text { Comercio }\end{array}$ & 802 & 86 & \\
$\begin{array}{l}\text { Resto } \\
\text { servicios }\end{array}$ & 773 & 100 & \\
\hline TOTAL & 3.413 & 385 & $4,80 \%$ \\
\hline
\end{tabular}

Fuente: Elaboración propia

* Estimada aplicando a la población de empresas promedio del $65 \%$ de empresas familiares

En la tabla 3, como en el caso anterior, se ha calculado la población de empresas familiares con 10 o más empleados por sectores de actividad. En este caso, para estimar la población de empresas familiares se ha usado el porcentaje global de $65 \%$ (ver tabla 1 ).
Adicionalmente, se ha comprobado que los datos recogidos son representativos de la población de acuerdo con la distribución sectorial de empresas: Se ha obtenido una correlación significativa del $0.835 \quad(p=0.01)$ entre el número de empresas en la población inicial y el número de empresas en la muestra final (esto es, las que han contestado de forma válida al cuestionario). Además, se ha desarrollado un análisis de la varianza, con el que no se han encontrado diferencias significativas entre el tamaño de las empresas de la población inicial y el de las empresas que han contestado de forma válida al cuestionario $(\mathrm{F}=0.332 ; \mathrm{p}=0.564)$.

\subsection{Variables y escalas}

\subsubsection{Formación universitaria del gerente}

Se han realizado diversos trabajos en la literatura de empresa familiar (Gallo et al., 2004; Galve y Salas, 2003), desde los que se ha identificado que existen diferencias significativas entre empresas familiares y no familiares, como consecuencia de la formación universitaria del gerente, resultando mucho menor, esta formación, en las empresas familiares. También se han detectado diferencias entre empresas familiares, identificando a la formación del gerente, como causante de las diferencias entre dichas empresas. Se trata de un factor importante desde el que explicar la capacidad de dirigir la empresa de un directivo (Wiersema y Bantel, 1992). La variable explica las prácticas desarrolladas en el negocio, y se codifica por medio de una variable dicotómica, al igual que otros estudios previos (Spicer, Dunfee, y Bailey, 2004), donde 1 implica formación universitaria del gerente, y 0 lo contrario.

\subsubsection{Potencial motivador del puesto.}

El estudio de la motivación en el trabajo es un aspecto tratado en diversas investigaciones. Así, Hackman y Oldman (1976), partiendo de la investigación de Turner y Lawrance (1965), desarrollan el modelo de características del 
puesto en el que se evalúa la motivación del puesto, a partir de cinco características del puesto: variedad de tareas, identidad de tareas, significación de tareas, autonomía y retroalimentación del puesto. Estas cinco características se combinan dentro de un sólo índice global que refleja el potencial de un puesto para fomentar la motivación, a este índice se le denomina Potencial Motivador del Puesto (PMP). Se han utilizado cinco ítems para medir, las características del potencial motivador del puesto, extraídos de la escala revisada de Idaszak y Drasgow (1987), a partir del trabajo de Hackman y Oldham (1980). Cada ítem se ha medido por medio de una escala likert de 5 puntos que varía desde 1 , o muy en desacuerdo, a 5, o muy de acuerdo, al igual que en otros estudios (ver Asgari et al., 2008). Los encuestados muestran para cada ítem su grado de acuerdo.

De la escala revisada por Idaszak y Drasgow (1987) se han extraído, los siguientes ítems, para examinar la motivación en el trabajo (ver tabla 4): "Indique su grado de acuerdo con las siguientes afirmaciones, con relación a la mayoría de los puestos de trabajo, en su empresa: “... necesitan utilizar complejas y variadas habilidades" (variedad), “...son responsables de la producción total de un producto, de un componente o de un servicio" (identidad), “... son muy significativos e importantes, ya que afectan a los resultados y al trabajo de otros puestos de la empresa" (significación), “... pueden actuar con libertad e independencia" (autonomía), “...conocen si el trabajo está bien realizado" (retroalimentación). Estas cinco variables, se ponen en relación a través de la siguiente fórmula: $\mathrm{PMP}=[(\mathrm{V}+\mathrm{I}+$ S) /3] x A x R

\subsubsection{Rendimiento}

Se ha utilizado la escala multidimensional de propuesta por Quinn y Rohrbaugh (1983), y se han incluido 4 ítems de éxito diferentes (ver tabla 4), correspondientes a cada una de las cuatro dimensiones propuestas por los autores, modelo de proceso interno (mejora en la calidad del producto), modelo de sistema abierto (incremento de la habilidad de adaptación a las necesidades cambiantes de los mercados), modelo racional (incremento de la productividad), finalmente, modelo de relaciones humanas (compromiso de los trabajadores). Tras comprobar la fiabilidad y validez de las escalas, se genero un indicador como media de los ítems de la escala. 
Tabla 4

Escalas de medida y análisis factorial confirmatorio.

\begin{tabular}{|c|c|c|c|}
\hline Variable/Ítems & $\lambda_{\text {c.e }}(\mathrm{t})$ & $\mathrm{R}^{2}$ & Fiabilidad \\
\hline \multicolumn{4}{|l|}{ Motivación laboral } \\
\hline \multirow{3}{*}{$\begin{array}{l}\text { La mayoría de puestos de trabajo en su empresa } \\
\ldots \text { necesitan utilizar complejas y variadas } \\
\text { habilidades } \\
\ldots \text { son responsables de la producción total } \\
\text { de un producto, de un componente o de un } \\
\text { servicio }\end{array}$} & & & $\varrho_{c}=0,84$ \\
\hline & $0,58(12,855)$ & 0,33 & a.v.e $=0,52$ \\
\hline & $0,52(13,118)$ & 0,27 & \multirow[t]{4}{*}{$\alpha=0,83$} \\
\hline $\begin{array}{l}\text {... son muy significativos e importantes, ya } \\
\text { que afectan a los resultados y al trabajo de } \\
\text { otros puestos de la empresa }\end{array}$ & $0,84(8,658)$ & 0,71 & \\
\hline $\begin{array}{l}\ldots \text { pueden actuar con libertad e } \\
\text { independencia }\end{array}$ & $0,76(11,025)$ & 0,58 & \\
\hline ...conocen si el trabajo está bien realizado & $0,84(8,845)$ & 0,70 & \\
\hline \multicolumn{4}{|l|}{ Rendimiento } \\
\hline $\begin{array}{l}\text { ¿Cómo considera la situación de su empresa respecto } \\
\text { de sus competidores?: }\end{array}$ & & & $\varrho_{c}=0,82$ \\
\hline Calidad del producto / servicio & $0,60(12,32)$ & 0,36 & a.v $\cdot e=0,54$ \\
\hline Adaptación al mercado & $0,75(10,19)$ & 0,56 & $\alpha=0,82$ \\
\hline Productividad & $0,81(8,35)$ & 0,66 & \\
\hline Compromiso de los trabajadores & $0,77(9,79)$ & 0,59 & \\
\hline
\end{tabular}

Bondad de ajuste del análisis factorial confirmatorio (CFA):

$\chi^{2}(27)=85.56(\mathrm{p}=0.000), \mathrm{NFI}=9,38, \mathrm{NNFI}=0,94, \mathrm{CFI}=0,96, \mathrm{IFI}=0,96, \mathrm{MFI}=0,93$,

$\mathrm{GFI}=0,95, \mathrm{AGFI}=0,92, \mathrm{RMR}=0,05, \mathrm{SRMR}=0,07, \mathrm{RMSEA}=0,07$

Valores recomendados para un buen ajuste de los datos: (Marcoulides y Schumacker, 1996; Hoyle y Panter,

1995; Premkumar y King, 1994)

$\chi^{2}(\mathrm{p}<0.05), \mathrm{NFI}>0,9, \mathrm{NNFI}>0,9, \mathrm{CFI}>0,9, \mathrm{IFI}>0,9, \mathrm{MFI}>0,9$,

Fuente: Elaboración propia

GFI $>0,9$, AGFI $>0,9$, RMR $<0,1$, SRMR $<0,1$, RMSEA $<0,06$

\subsection{Fiabilidad y validez de las escalas utilizadas}

La evaluación del modelo de medida implica el análisis de la fiabilidad individual del ítem, la consistencia interna o fiabilidad de una escala, el análisis de la varianza extraída media y la validez discriminante (ver tabla 4). Se ha efectuado, para ello, un análisis factorial confirmatorio (CFA), mediante el método de estimación de máxima verosimilitud, ya que es un método más adecuado cuando no se da la normalidad de los datos (Chou et al.,1991; Hu et al., 1992; Bentler, 1995; West et al., 1995), como es el caso.

Se han utilizando los índices propuestos por Fornell y Larcker (1981) y Bagozzi y Yi (1988) de varianza extraída (a.v.e) y fiabilidad

compuesta ( $\rho c)$ respectivamente. En este caso, el método de ofrece indicadores similares de

ajuste, más restringidos, que el resto de métodos (Bentler, 1990), utilizando la distribución Chi Cuadrado del estadístico de Satorra-Bentler (Satorra y Bentler, 1991), tal y como sugiere la literatura (Hair et al., 1999; West et al., 1995). La calidad del ajuste es la correspondencia existente entre la matriz de varianzascovarianzas observada y la matriz estimada (de medida). Gracias al CFA podemos evaluar de forma simultánea la multidimensionalidad y la fiabilidad de la medida de cada constructo y dimensión. Sobre la base de los resultados obtenidos $^{1}$ podemos concluir que el modelo es

1. Las medidas de ajuste se han estimado utilizando EQS v. 6.1. Los
indicadores están dentro de los parámetros recomendados por la
literatura (Marcoulides y Schumacker, 1996; Hoyle y Panter, 1995;
Premkumar y King, 1994), por lo que existe un buen ajuste en el modelo
de medida $(\chi 2(27)=85.56(\mathrm{p}=0.000)$, Bentler-Bonet normed fit index
$[\mathrm{NFI}]=9,38$, Bentler-Bonet non-normed fit index [NNFI] $=0,94$,
Comparative fit index [CFI] $=0,96$, Bollen's fit index [IFI] $=0,96$,
McDonald's fit index [MFI] $=0,93$, Joreskog-Sorbom's fit index [GFI]
0,95 , Joreskog-Sorbom's fit index [AGFI] $=0,92$, Root mean square
residual [RMR] $=0,05$, Standarized root mean square residual Carrasco Hernández, A. J. y Meroño Cerdan, A. L. (2011). Efectos de la formación universitaria del gerente de pymes familiares en la motivación laboral del empleado. Revista de Empresa Familiar, 1(1), pag. 35-51. 
adecuado para medir los constructos especificados.

En cuanto a la fiabilidad o consistencia interna de cada escala podemos apreciar que todas las escalas presentan un valor adecuado de fiabilidad compuesta $\left(\rho_{c}\right)$, tal y como proponen Fornell y Larcker (1981) y Bagozzi y Yi (1988), al ser superiores todos a 0,8 (recomendable mayor de 0,6).

Por lo que respecta a la varianza extraída (AVE), todas las escalas ofrecen un valor superior al límite recomendable de 0,5. Los resultados se presentan en la tabla 4. También los indicadores $\alpha$ de Crombach confirman la alta fiabilidad de las escalas, con valores superiores a 0,8 (Hair et al., 2006).

Finalmente, ha sido comprobada la validez del modelo a partir de la validez de contenido y la validez de constructo.

La primera de ellas viene avalada por la revisión bibliográfica efectuada en los epígrafes anteriores, la cual ha servido para definir los conceptos que se han contrastado en el estudio, mientras que para comprobar la validez de constructo debe analizarse tanto la validez convergente como la discriminante (Tabla $4 \mathrm{y}$ 5).

Respecto a la validez convergente, hace referencia a la convergencia de cada indicador con el factor que conforma, comprobándose ésta a través del valor de cada uno de los ítemes (como mínimo debe ser superior a 0.5) y de su significatividad $(\mathrm{t}>1,96)$ (Hildebrant, 1984). Igualmente, se comprobó la validez discriminante, a través del cálculo del intervalo de confianza para cada par de factores los de confianza (+/- 2 veces el error estándar).

De este modo, se ha comprobado que ninguno de ellos contiene el valor $1 \mathrm{y}$ que, por lo tanto, las dimensiones son significativamente diferentes (Peter, 1981; Anderson y Gerbing, 1988).

A la vista de los resultados, el modelo de medida resulta adecuado, por lo que nos encontramos en disposición de contrastar las hipótesis planteadas en este trabajo.

\section{Resultados}

4.1. Análisis descriptivo: características de las empresas de la muestra.

El estudio se ha realizado sobre una muestra de 385 pymes familiares, en las que 124 pyme tienen un gerente con formación universitaria (esto es, un 32,20\%) y 261 pyme tienen un gerente sin formación universitaria (esto es, $67,80 \%)$.

En la tabla 5 se puede ver un resumen de los valores medios de diversas variables que describen rasgos significativos de las empresas del estudio (\% derechos de voto familiares, \% directivos familiares, número de accionistas, $\mathrm{n}^{\circ}$ de empleados, antigüedad de la empresa, total activos, ingresos de explotación, gastos de personal, resultados del ejercicio, fondos propios, margen sobre ventas, liquidez, solvencia y beneficios por empleado), en función de la formación del gerente.

[SRMR] $=0,07$, Root mean square error of aproximation [RMSEA]=0,07). Los estadísticos NFI, NNFI, CFI, IFI, MFI, GFI, AGFI son superiores a 0,9 , RMR y SRMR son inferiores a 0,1 , y RMSEA es inferior a 0,08 , tal y como se recomienda en la literatura. Si nos detenemos en el análisis de los indicadores individuales para cada ítem, todos presentan valores significativos del coeficiente estandarizado a un nivel del $95 \%(\mathrm{p}<0,05)$. 
Tabla 5

Estadísticos descriptivos.

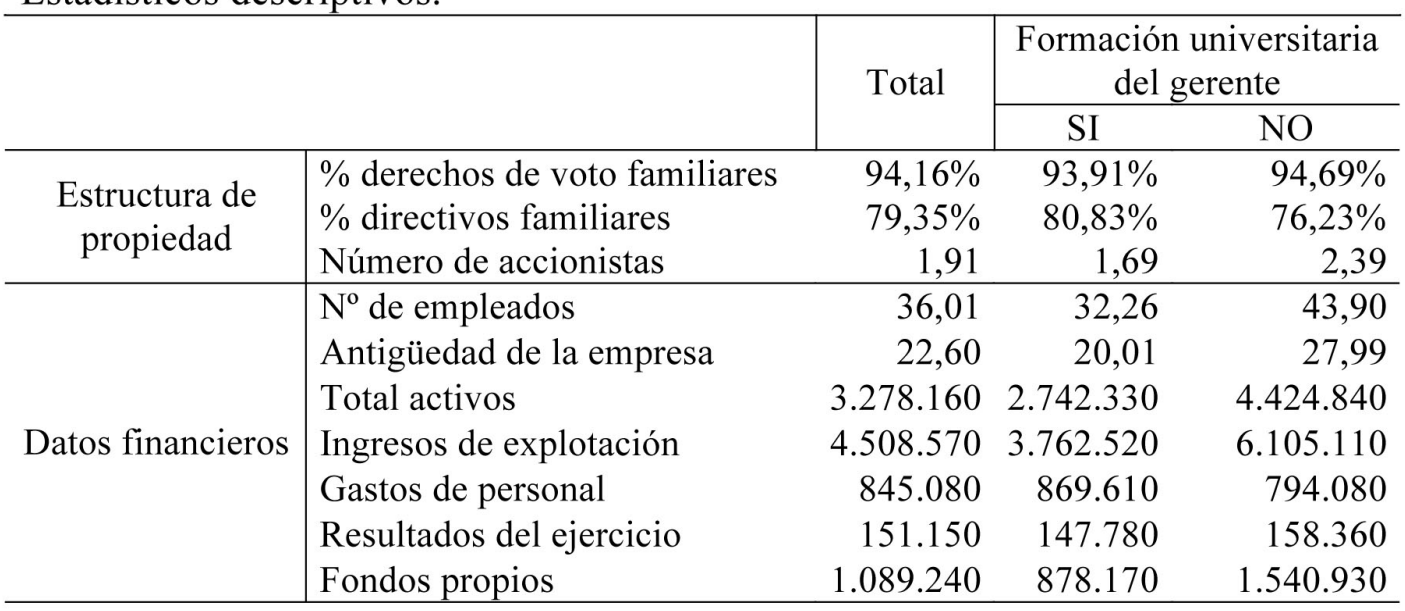

En primer lugar, examinando la estructura de propiedad de las pyme estudiadas, se observa una alta presencia de la familia en la propiedad $(94,16 \%)$, no existiendo con relación a la concentración de la propiedad, grandes diferencias entre los grupos de empresas. El número medio de accionistas en las pyme examinadas es de 1,91 accionistas, siendo mayor en empresas con gerente con formación universitaria (2,39 accionistas) frente a las empresas con gerente sin formación universitaria (1,69 accionistas). La presencia de la familia en los órganos de poder, esto es, el porcentaje de directivos familiares es del (79,35\%), aunque disminuye en las pyme con gerente con estudios universitarios $(76,23 \%$ frente a $80,83 \%$ ).

El tamaño medio y la antigüedad media de las empresas examinadas es de 36,01 empleados y 22,60 años, siendo mayor en las empresas de gerente con formación universitaria $(43,9$ empleados y 27,99 años frente a 32,26 empleados y 20,01 años). De igual modo, el tamaño de la empresa por activos, los ingresos de explotación, los fondos propios y los resultados del ejercicio son mayores en las empresas de gerente con formación universitaria. No así, los gastos de personal, que son mejor administrados en las pyme de gerente con formación universitaria (794.080 euros) que en las que no (845.080 euros).

\subsection{Análisis explicativo: prueba t y regresión} lineal.

Con el objetivo de diferenciar qué características del puesto explican las diferencias entre pyme familiares, en función de si el gerente posee o no formación universitaria, se ha efectuado un contraste de medias, para ello, se ha realizado una prueba $t$ de muestras independientes (ver tabla 6). Éste permite conocer las diferencias significativas entre pymes, para cada una de las 5 características del puesto evaluadas en el modelo de Hackman y Oldman (1976), así como para el potencial motivador del puesto.

Por lo que respecta al potencial motivador del puesto y a las cinco características del puesto que lo definen (ver tabla 6), los resultados del análisis muestran que existen diferencias significativas entre las pyme dirigidas por un gerente con formación universitaria $(49,97)$ frente a las pyme dirigidas por un gerente sin formación universitaria $(35,80)$. 


\section{Tabla 6}

Medias y test univariantes.

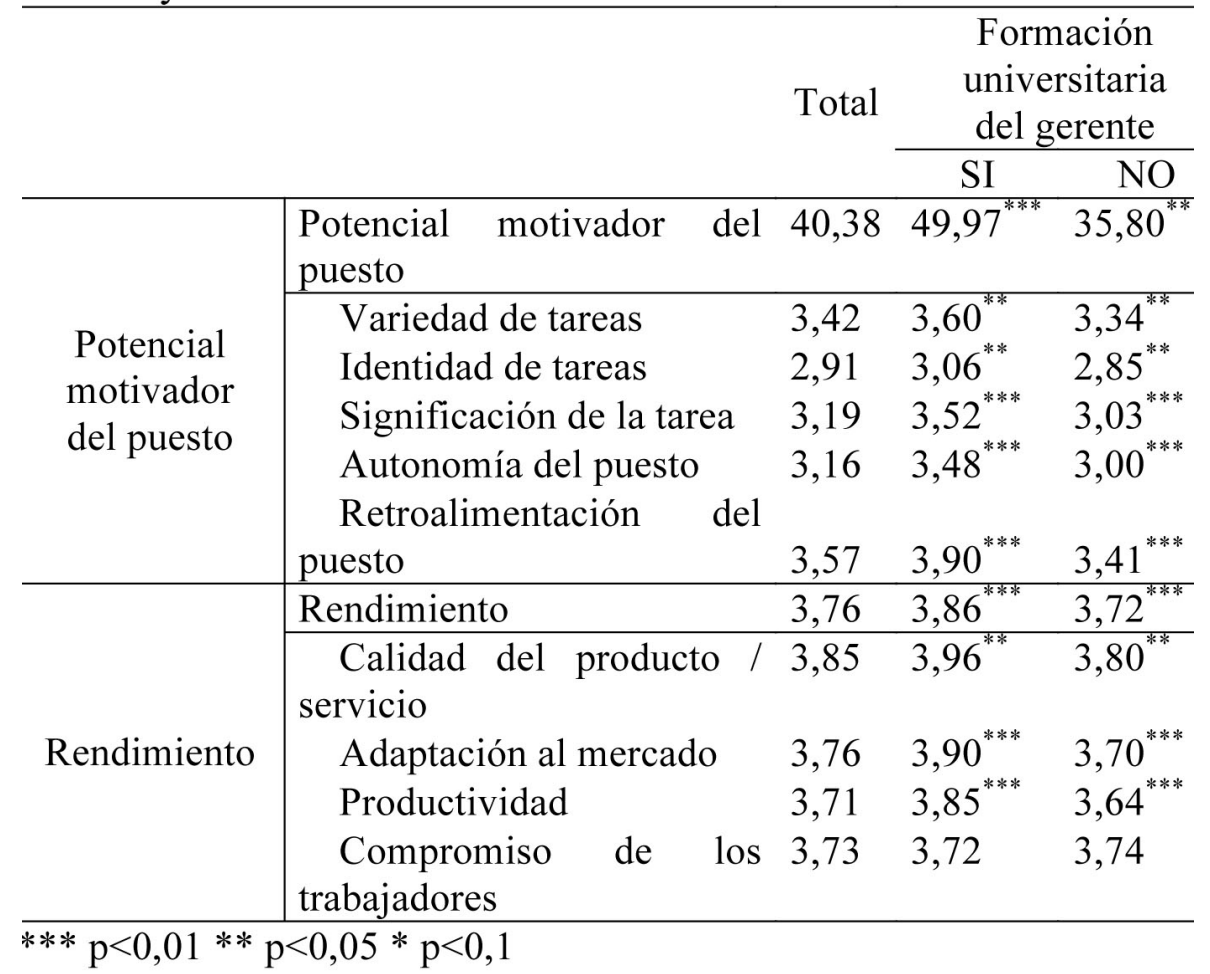

Las pymes dirigidas por un gerente con formación universitaria se caracterizan por tener empleados (ver figura 1) que en su puesto disponen de una mayor variedad de tareas, identidad de tareas, significación de la tarea, autonomía del puesto y retroalimentación del puesto. Por lo que no encontramos evidencias empíricas, con las que rechazar la hipótesis nula $1\left(\mathrm{H}_{1}\right)$. Se acepta, por tanto, la hipótesis 1 como válida.

En segundo lugar, se muestran las diferencias obtenidas para la variable rendimiento (ver tabla 1 y figura 2). Los resultados obtenidos permiten sostener que, en general, las empresas dirigidas por un gerente con formación universitaria presentan un mayor rendimiento que las empresas dirigidas por un gerente sin formación universitaria. Tan sólo no existen diferencias significativas, en el compromiso de los empleados, al comparar ambos grupos de pyme. A priori, parece existir una relación positiva, entre el rendimiento y el potencial motivador del puesto, al poseer el gerente formación universitaria.

Para contrastar la hipótesis segunda y corroborar los resultados anteriores, se ha realizado un análisis de regresión jerárquica, cuyos resultados aparecen en la tabla 8 .

\section{Tabla 8}

Análisis de regresión jerárquica.

\begin{tabular}{l|l}
\hline & $\begin{array}{l}\text { Modelos / } \\
\text { Rendimiento }\end{array}$ \\
\cline { 2 - 2 } & 1 \\
\hline
\end{tabular}

\section{Potencial}

Motivador puesto (PMP)

Formación del

gerente $(\mathrm{F}) \quad 0,11^{* *} \quad 0,10^{* * *}$

Formación x PMP $\quad 0,05$

\begin{tabular}{lll}
\hline $\mathrm{R}^{2}$ & 0,040 & 0,042 \\
\hline $\mathrm{F}$ & $7,574^{* * *}$ & $5,225^{* * *}$ \\
\hline$\Delta \mathrm{R}^{2}$ & 0,02 \\
\hline$* * * \mathrm{p}<0.01 ; * * \mathrm{p}<0.05 ; * \mathrm{p}<0.1$ &
\end{tabular}

En la tabla 7, se adjuntan los estadísticos descriptivos y las correlaciones de los constructos del estudio. Como se puede observar, existen correlaciones significativas entre las variables de rendimiento, potencial motivador del puesto y formación, tal y como se esperaba en la literatura académica, si bien, ninguna es excesivamente alta, lo que indica 
que no existen problemas de multicolinealidad corroborado mediante los índices de tolerancia.

\section{Tabla 7}

Correlaciones y estadísticos descriptivos.

\begin{tabular}{llllll}
\hline & Media & D.T & 1 & 2 & 3 \\
\hline $\begin{array}{l}\text { Formación } \\
\text { del gerente }\end{array}$ & 0,32 & 0,47 & 1 & \\
\hline P.M.P & 41,18 & 25,15 & $0,28^{* * *} 1$ & \\
\hline Rendimiento & 3,80 & 0,46 & $0,11^{* *} 0,21^{* * *} 1$ \\
\hline$* * * \mathrm{p}<0,01 * *$ & $\mathrm{p}<0,05 * \mathrm{p}<0,1$ & &
\end{tabular}

Se han elaborado dos regresiones, con el rendimiento de la empresa como variable dependiente, y el potencial motivador del puesto y la formación del gerente como variables independientes.

Con la primera regresión se añade el potencial motivador del puesto y la formación del gerente, y con la segunda regresión se añade el efecto de interacción entre el potencial motivador del puesto y la formación del gerente. En este sentido, en términos generales, se produce un incremento del poder explicativo en los modelos de rendimiento una vez introducidas las variables de potencial motivador del puesto y formación, no así, al introducir el efecto de interacción entre el potencial motivador del puesto y la formación (modelo 1: $\Delta \mathrm{R}^{2}=0.04, \mathrm{~F}=7,574, \mathrm{p}<0.01$; modelo 2: $\left.\Delta \mathrm{R}^{2}=0.01, \mathrm{~F}=5.225, \mathrm{p}<0.01\right)$.

Contrastando específicamente la hipótesis segunda, los resultados para el rendimiento indican que existen diferencias significativas entre empresas dirigidas por un gerente con formación universitaria frente a las que son dirigidas por un gerente sin formación universitaria (modelo 1, $\beta=0.11, \quad \mathrm{p}<0.05$; modelo $2, \beta=0.10, p<0.05$ ), al igual que también existen un efecto significativo del potencial motivador del puesto en el rendimiento (modelo $1, \beta=0.14, p<0.05$; modelo $2, \beta=0.11, p<0.05)$.

Sin embargo, la interacción entre el potencial motivador del puesto y la formación del gerente no produce efectos significativos positivos en el rendimiento de la empresa (modelo $2, \beta=0.11$, $\mathrm{p}>0.1$ ), lo que nos lleva a rechazar la hipótesis 2 .

\section{Conclusiones}

El propósito de este trabajo ha sido examinar la relación entre la formación del gerente y la motivación en el puesto de trabajo de los empleados en las pyme familiares, encontrando que el potencial motivador de los puestos en las pyme familiares con gerentes formados universitariamente es mayor que el de las pyme familiares con gerentes no formados universitariamente. Tales diferencias, aparecen en todas y cada una de las características que definen el puesto. Así, siguiendo el modelo de Hackman y Oldman (1976), encontramos diferencias entre ambos grupos de pyme en variedad de tareas, identidad de tareas, significación de tareas, autonomía y retroalimentación del puesto, estando definidas de forma más amplia en la pyme con gerentes formados universitariamente. En concreto, la autonomía y la retroalimentación son las que mayor peso y efectos directos tienen sobre el potencial motivador del puesto.

Estos hallazgos pueden servir para explicar por qué en la actualidad existen problemas de reclutamiento de personal cualificado en tales organizaciones, al resultar las pyme familiares menos atractivas para aquellos empleados más formados y con mayor potencial. A menudo estos trabajadores suelen rechazar las ofertas de trabajo en este tipo de empresas por el hecho de sentirse infravalorados por sus directivos, y al sentir que no existen posibilidades de promoción en ellas. Además de sentir que los gerentes en tales organizaciones, poseen un menor grado de formación que sus homólogos en otras empresas más profesionalizadas (Schulze et al., 2001), lo que conduce a la pérdida de motivación laboral por parte de los empleados.

Por otro lado, no se ha encontrado un efecto de mejora en el rendimiento de la empresa por la interacción entre el potencial motivador del puesto y la formación del gerente. Hallazgo sorprendente, que denota, que los gerentes formados, de pyme familiares, son incapaces de aprovechar el mayor potencial motivador de los puestos de los empleados para alcanzar un mayor rendimiento con sus pyme, en 
comparación con el rendimiento alcanzado por los gerentes sin formación universitaria de pyme familiares.

Una explicación para este hallazgo, reside en la implicación de la familia en la empresa, y es que a pesar de que se forma universitariamente al gerente, no se le deja total libertad de actuación en el negocio. La mayor formación del gerente se ve contrarrestada por los valores, la cultura, la tradición, el poder y la experiencia de la familia. Esto es, lo que en pyme familiar se define como Familiness (Habbershon, Williams, y MacMillan, 2003; Chrisman, Chua y Steier, 2005), o recursos generados por la interacción de la familia en el negocio. Estos recursos, pueden tener efectos positivos al combinarse con otros recursos empresariales en el rendimiento de la empresa, y generar ventajas competitivas, o todo lo contrario, producir efectos negativos y desventajas competitivas en el negocio familiar (Hoelscher, 2002; Hoffman et al., 2006). Falta por tanto, que la mayor formación del gerente en la pyme familiar, sea mejor aprovechada al ser combinada con otros recursos de la empresa para generar ventajas competitivas, más cuando se combine con los recursos procedentes de la familia, para generar capital familiar, el recurso empresarial estratégicamente más importante para la pyme familiar (Hoffman et al., 2006).

En definitiva, se ha comprobado que la formación del gerente conduce a un mayor potencial motivador del puesto, que el potencial motivador del puesto conduce a un mayor rendimiento en la empresa, pero que las pyme familiares no saben combinar formación del gerente y potencial motivador del puesto para alcanzar ventajas competitivas sostenibles.

No obstante, como se ha visto, el estudio del potencial motivador del puesto es complejo y necesita ser tratado en futuras investigaciones, en las que es preciso, por un lado, introducir otras variables que pueden influir de manera significativa en las relaciones motivación laboral del empleado-formación - como las prácticas de formación en la empresa dirigidas a empleados, el grado de participación de la familia en las estructuras de poder del negocio, la experiencia familiar, la cultura organizativa, entre otras- y, por otro lado, superar limitaciones como las que presenta el trabajo actual, derivadas fundamentalmente de que los datos y escalas no son ad-hoc, es decir, manejamos datos de corte transversal que están limitados por un espacio y momento temporal concreto. Por otro, las escalas son apreciaciones humanas, pero no son totalmente objetivas. Los datos son de la Región de Murcia y no sabemos con certeza si se puede extrapolar al resto de comunidades españolas y países. La escala de formación del gerente podría ser mejorada si se distinguen qué tipo de estudios universitarios posee el gerente, y en caso de no poseerlos, si se recoge si dispone o no de formación profesional. El valor de los datos se enriquecería mucho, si la recogida de información se realizará para otros directivos y empleados, diferentes al gerente, sean estos, familiares o no. Por último, es una limitación actual, la no inclusión de variables de control y moderadoras, tales como el tamaño, sector de actividad o edad de la empresa. Aunque, todos estos aspectos, podrán ser mejorados en un futuro próximo con nuevos trabajos, en esta línea.

\section{Bibliografía}

Ahire. S. L. y Golhar, D. Y (1996). Quality Management in Large versus Small Firms. Journal of Small Business Management, 34(2), $1-11$.

Amit, R. y Schoemaker, P. (1993). Strategic Assets and Organizational Rent. Strategic Management Journal, 14(1), 33-46.

Anderson, J.C. y Gerbing, D. (1988). Structural Modellimg in Practice: A Review and Recommended Two-Steps Approach. Psichological Bulleting, 103(3), 411-423.

Aragón, A. y Rubio, A. (2005). Factores asociados con el éxito competitivo de las Pymes en España. Universia Business Review, 8(3), 3851.

Asgari, A., Silong, A.D., Ahmad, A. y Samag, B.A. (2008). The Relationship between Organizational Characteristics, Task Characteristics, Cultural Context and 
Organizational Citizenship Behaviors. European Journal of Economics, Finance and Administrative Sciences, 13, 94-107.

Astrachan, J. y Shanker, M. (2003). Family Businesses' Contribution to the U.S. Economy: A Closer Look. Family Business Review, 16(3), 211-219.

Bagozzi, R. y YI, Y. (1988). On the evaluation of structural equation models. Academy of Marketing Science, 16(1), 74-94.

Bantel, K. y A, Jackson, S. E (1989). Top Management and Innovations in Banking: Does the Composition of the Top Team Make the Difference? Strategic Management Journal, 10(2), 107-124.

Barney, J. (1986). Organizational culture: Can it be a source of sustained competitive advantage? Academy of Management Review, 11(3), 656665.

Barney, J. (1991). Firm resources and sustained competitive advantage. Journal of Management, 17(1), 99-120.

Barney, J. B. y Wrigth, P. M. (1998). On becoming a strategic partner: The role of human resources in gaining competitive advantage Human Resource Management, 37(1), 31-46.

Barney, J.B. y Arikan, A.M. (2001). The resource-based view: origins and implications en M.A. HITT, R.E. Freeman y J.S. Harrison, J.S. (Eds.) The Blackwell Handbook of Strategic Management, Oxford: Blackwell.

Bentler, P.M. (1990). Comparative fit indexes in structural models. Psychological Bulletin, 107(2), 238-246.

Bentler, P.M. (1995). EQS structural equations program manual. Encino, California: Multivariate Software.

Cabrera-Suárez, K., Saá-Pérez, D. y GarcíaAlmeida, D. (2001). The Succession Process from a Resource and Knowledge-Based View of the Family Firm, Family Business Review, 14(1), 37-47.

Chou, C.P., Bentler, P.M. y Satorra, A. (1991). Scaled Test Statistic and Robust standard Errors for Non-normal Data in Covariance Structure
Analysis, British Journal of Mathematical and Statistical Psychology, 44(2), 347-357.

Davis. P. y Harverston, P. (2000). Internationalization and organizational growth: the impact of Internet Usage and Technology involvement among enterpreneur-led family business. Family Business Review, 13(2), 107120.

De Kok, J.M.P., Uhlaner, L.M. y Thurik, A.R. (2006). Professional HRM practices in family owned-managed enterprises. Journal of Business Management, 44(3), 441-460.

Drucker, P. (2002). La Gerencia. Buenos Aires: El Ateneo.

Dyer, W. G. (2006). Examining the "Family Effect" on Firm Performance. Family Business Review, 19(4), 253-273.

Fernández, Z. y Nieto, M. J. (2005). Internationalization Strategy of Small and Medium-Sized Family Businesses: Some Influential Factors. Family Business Review, 18(1), 77-89.

Fernandez, Z. y Nieto, M.J. (2006). Impact of ownership on the international involvement of SMEs. Journal of International Business Studies, 37(3), 340-351.

Fornell, C. y Larcker, D. F. (1981). Evaluating structural equation models with unobservable variables and measurement error: algebra and statistics. Journal of Marketing Research, 38(1), 39-50.

Gallo, M., Ariño, A., Máñez, I. y Cappuyns K. (2004). La internacionalización a través de alianzas estratégicas en las empresas familiares. Navarra: Universidad de Navarra, IESE.

Galve, C. y Salas, V. (2003). La empresa familiar en España. Fundamentos económicos y resultados. Bilbao: Fundación BBVA.

Habbershon, T.G, Williams. M. y MacMillan. I.C (2003). A unified systems perspective of family firm performance. Journal of Business Venturing, 18(4), 451-465.

Habbershon, T.G. y Williams, M. (1999). A resource-based framework for assessing the 
strategic advantage of family firms. Family Business Review, 12(1), 1-25.

Hackman, J. R. y Oldham, G. R. (1976). Motivation through the design of work: test a theory. Organizational Behavior and Human Performance, 16(2), 250-279.

Hair, J., Anderson, R. y Black, W. (1999). Análisis Multivariante, Madrid: Prentice Hall.

Hitt, M. Ireland, R. D., Camp, S. M. y Sexton, D. L. (2001). Strategic entrepreneurship: entrepreneurial strategies for wealth creation. Strategic Management Journal, 22(6-7), 479491.

Hitt, M., Ireland, R. D., Camp, S. M. y Sexton, D. L. (2002). Strategic entrepreneurship: Integrating entrepreneurial and strategic management perspectives. En Hitt, M., Ireland, R. D., Camp, S. M. y Sexton, D. L. Strategic entrepreneurship: creating a new mindset. Oxford: Blackwell Publishing.

Hoelscher, M. L. (2002). The relationship between family capital and family business performance: Collaboration and conflict as moderators. Ph.D. Dissertation, Texas Tech University, Texas, EEUU.

Hoffman, J., Hoelscher, M. y Sorenson, R. (2006). Achieving Sustained Competitive Advantage: A Family Capital Theory. Family Business Review, 1(2), 135-145.

Hu, L., Bentler, P.M. y Kano, Y. (1992). Can test Statics in covariance structure Analysis be Trusted?, Psychological Bulletin, 112(2), 351362.

Idaszak, J. R, y Drasgow, F. (1987). A revision of the Job Diagnostic Survey: Elimination of a measurement artefact, Journal of Applied Psychology, 72(1), 69-74.

Kellermanns, F. W. (2005). Family Firm Resource Management: Commentary and Extensions. Entrepreneurship Theory and Practice, 29(3), 313-319.

Klein, S., Astrachan, J. y Smyrnios, K. (2005). The F-PEC scale of family influence: Construction, validation and further implication for theory, Entrepreneurship Theory \& Practice, 29(3), pp. 321-339.
Lerner. M. y Almor. T. (2002). Relationships among Strategic Capabilities and Performance of owned small ventures. Journal of Small Business Management, 40(2), 109-125.

Mahoney, J. T. (1995). The management of resources and the resource of management. Journal of Business Research, 33(2), 91-101.

Makadok, R. (2001). Toward a synthesis of the resource-based and dynamic-capability view of rent creation. Strategic Management Journal, 22(5), 387-401.

Malik, F. (2000). Job Design: Diseño del puesto de trabajo. Revista Capital Humano, 119(13). Madrid.

Martin, G. y Staines. H. (1994). Managerial Competences in Small Firms, The Journal of Management Development, 12(7), .23-34.

Monreal, J., Sánchez Marín, G., Meroño Cerdán, A. y Sabater Sánchez, R. (2009). La gestión de las empresas familiares: un análisis integral. Madrid: Thomson-Civitas.

Noe, R. (1997). Administración de Recursos Humanos. México: Ed. Prentice Hall Hispanoamericana.

Norburn, D. y Birley (1988). The Top Management Team and Corporate Performance. Strategic Management Journal, 9(3), 225-237.

Penrose, E. (1959). The Theory of Growth of the Firm. Oxford: Basil Blackwell.

Priem. R. y Butler, J. (2001). Is the resourcebased view a useful perspective for strategic management research?, Academy of Management Review, 26(1), 22-40.

Quinn, R.E. y Rohrbaugh, J. (1983). A spatial model of effectiveness criteria: towards a competing values approach to organizational analysis. Management Science, 29(3), 363-377.

Reid. S. y Adams, J. (2001). Human Resource Management a Surrey of practices within family and non-family firms. Journal of European Industrial Training, 25(6), 310-320.

Rubio, A. y Aragón, A. (2009). Recursos críticos y estrategia en la pyme industrial. Información Comercial Española, ICE: Revista de economía, 846(1), 193-212. 
Rubio, A. (2004). Factores explicativos del éxito competitivo en la PYME, Tesis Doctoral, Universidad de Murcia, Murcia, España.

Satorra, A. y Bentler, P.M. (1991). Goodness of fit test under IV estimation: Asymtotic robustness of a NT test statistic. En Gutierrez, R. y M.J. Valderrama (Eds.), Applied Stochastic Models and Data Analysis (pp. 555-567). London: World Scientific.

Schein, E. (1982). Psicología de la Organización. México: Prentice Hall.

Schulze, W.S., Lubatkin, M.H., Dino, R.N. y Buchholtz, A.K. (2001). Agency relationships in family firms: Theory and evidence. Organizational Science, 12(2), 99-116.

Shanker, M., y Astrachan, J. (2003). Family Businesses' Contribution to the U.S. Economy: A Closer Look. Family Business Review, 16(3),
211-219.

Turner, P. y Lawrence. R. (1965). Industrial Jobs and the Worker. Boston: Harvard Business School.

Wernerfelt, B. (1984). A Resource-based View of the Firm, Strategic Management Journal, 5(2), 171-180.

West, S.G., Finch, J.F. y Curran, P.J. (1995). Structural equation models with non-normal variables: Problems and remedies, in Hoyle, R.H (Eds), Structural equation modeling: Concepts, issues and applications. California: Sage Publications.

Wiersema M. F. y Bantel K. A. (1992). Top Management Team Demography and Corporate Strategic Change. Academy of Management Journal, 35(1), 91-121. 